\title{
Originalien
}

Notfall Rettungsmed 2021 · 24 (Suppl 1):S15-S20 https://doi.org/10.1007/s10049-020-00807-3 Accepted: 28 October 2020

Published online: 2 December 2020

(c) Springer Medizin Verlag GmbH, ein Teil von Springer Nature 2020

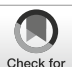

İshak Şan ${ }^{1}$ Burak Bekgöz ${ }^{2}$ Eren Usul ${ }^{3}$. Çağdaş Yıldırım ${ }^{4}$ Emin Gemcioğlu • Ahmet Fatih Kahraman ${ }^{4}$. Ahmet Emre Ay ${ }^{4}$

' Faculty of Medicine, Department of Emergency Medicine, University of Health Sciences, Ankara, Turkey

${ }^{2}$ Department of Emergency Medicine, Ankara City Hospital, Ankara, Turkey

${ }^{3}$ Emergency Service, Sincan State Hospital, Ankara, Turkey

${ }^{4}$ Faculty of Medicine, Department of Emergency Medicine, Yıldırım Beyazıt University, Ankara, Turkey

${ }^{5}$ Department of Internal Medicine, Ankara City Hospital, Ankara, Turkey

\section{Role of lung ultrasonography in the diagnosis of COVID-19 patients admitted to the emergency department}

patients may also present with diarrhea, nausea, and vomiting [5]. Although most patients experience mild disease, $14 \%$ of patients have lung involvement [6]. Lung imaging is therefore recommended for COVID-19 patients. The diagnostic value of chest radiography in the early stages is low in some lung disease [7]. However, lung computed tomography (LCT) can show lung involvement even before symptoms begin [8]. Although LCT represents the gold standard for the examination of most pulmonary diseases, it presents some important challenges, such as the high dose of radiation administered to the patient, its nonadministration to some groups of patients (e.g., pregnant women), the need to transport the patient to the radiology department, and the lack of CT scanning in some emergency departments (EDs) [7]. The American College of Radiology (ACR) recommends that CT not be used to screen for, or as a first-line test to diagnose, COVID19. The ACR also recommends that CT be used sparingly and be reserved for hospitalized, symptomatic patients with specific clinical indications for CT [9].

Ultrasonography (US), which is a practical and nonhazardous diagnostic tool, has recently been recognized as offering an efficient way of accurately diagnosing thoracic pathologies that cause dyspnea. In addition, lung ultrasound
(LUS) is a diagnostic method that can be applied quickly at the bedside and provide rapid results, without the patient needing to be transported to another location such as a CT room [10]. Lung ultrasound has been reported to provide more accurate results compared to methods such as physical examination and chest radiography, while for some diagnostic questions, it provides results nearly equivalent to CT [11]. Furthermore, LUS is more advantageous because it can be applied at the bedside. There is also no risk of ionizing radiation, and it can be applied easily by emergency physicians who can interpret the findings with the physical examination of the patients [12].

Although there have been suggestions that LUS can be used to detect the lung findings of COVID-19 patients, this information has been obtained from a small number of studies and case reports.

In this study, the capacity of LUS for the diagnosis of lung findings was evaluated in patients with suspected COVID19 who were admitted to the ED.

\section{Methods}

This observational clinical study was conducted in the ED of the Ankara City Hospital, which was a pandemic hospital during the period April 1-30, 2020. The study was approved by the Ethics Comthroat, and 

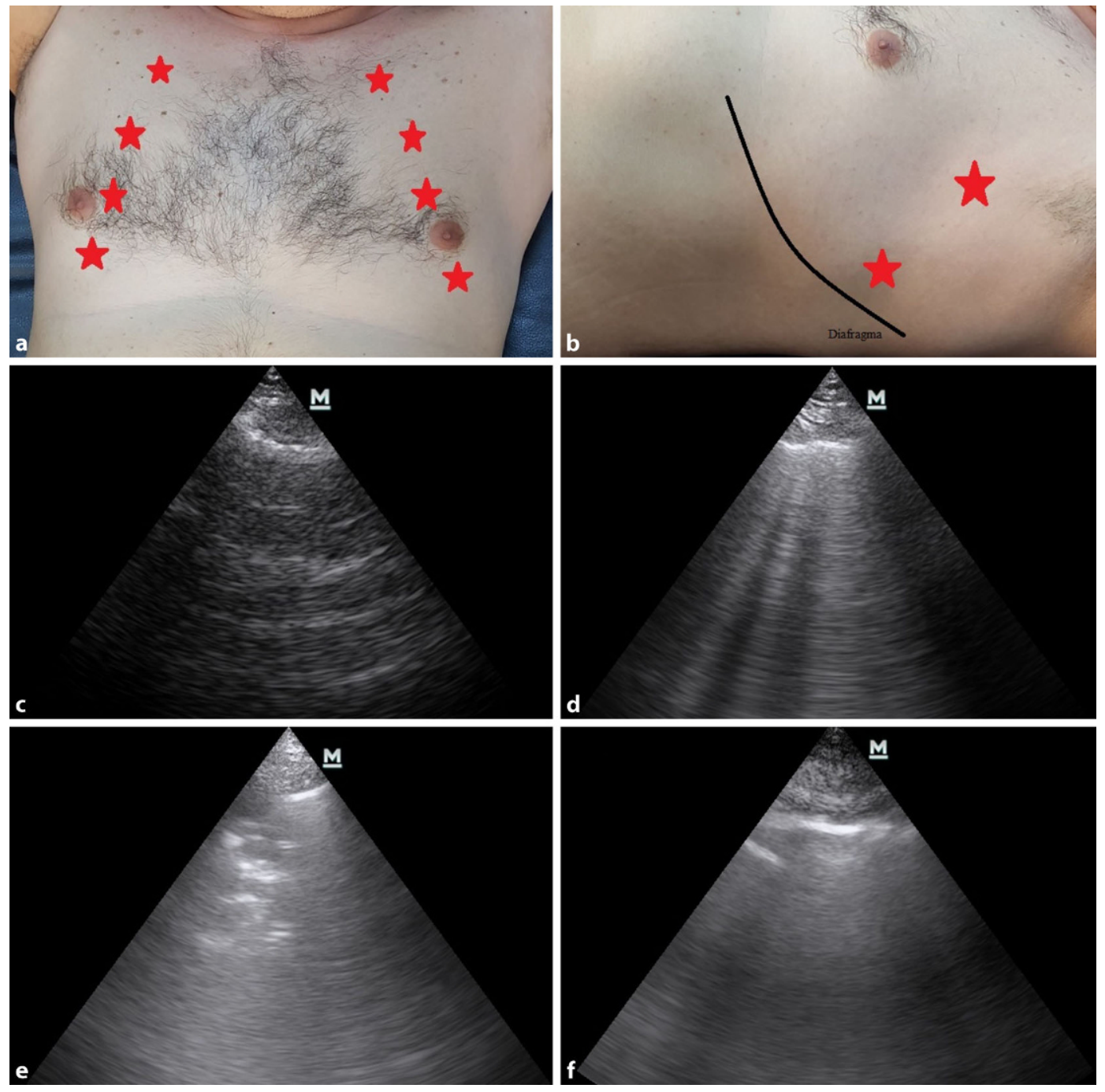

Fig. $1<$ a Anterior ultrasound points, b lateral ultrasound points, $c$ normal lung ultrasound, $\mathrm{d} B+$ lines, e lung consolidation and aerobronchograms, f pleural thickening

mittee No. 1 of the Ankara City Hospital (approval number E1-20-616).

Patients admitted to the ED, were triaged as COVID-19 infected, and then opted for tomography were included in the study. Those who were not triaged as COVID-19 infected, decided not to have LCT, or were vitally unstable on arrival were excluded from the study. The inclusion criterion was not to select patients consecutively admitted to the ED.

Patients with cough, shortness of breath, and a temperature exceeding $38^{\circ} \mathrm{C}$ were transferred from the triage area to the negative pressure area reserved for COVID-19 patients and provided with a mask. After evaluating their vital signs and conducting a physical examination, patients with suspected pneumonia were recommended for LCT but underwent LUS before LCT. Lung ultrasound was performed by 2 emergency physicians who had previously received basic and advanced US training and had at least 3 years' ED experience. All the physicians entering the COVID19 area for LUS took protective measures by wearing personal protective clothing, N95 mask, glasses, and gloves.

During LUS, longitudinal examinations were performed using a microconvex probe set at a frequency of $2-6 \mathrm{MHz}$ (Mindray M7, Guangdong, China). While applying LUS, the patients were placed in the supine or semirecumbent position, and each hemithorax was examined based on a total of 6 points: 4 anterior and 2 lateral (• Fig. 1a, b). Evaluation of the pleura was performed with high frequency because it was very difficult with low and medium fre- quency. After each LUS examination, the ultrasound probe was cleaned with hydrogen peroxide, and the new patient was examined with a clean probe.

During the LUS examination, the pleura between the two ribs was identified first. After detecting the pleura, the lung sliding sign, which is a normal finding that occurs as a result of the movement of the visceral and parietal pleura against each other, was evaluated. In the patients with lung sliding, $A$ and B lines were checked. A lines, which are reverberation artifacts of the pleura, were accepted as normal lung findings (- Fig. 1c). On the other hand, B lines, which originate from the pleura, appear as hyperechoic lines in the form of a beam that continues to the end of the screen, erasing A lines and shifting with the movement of the pleura. One or two 
B lines can be seen in each quadrant of each normal lung, but it is always pathological if there are $\geq 3$ in 1 quadrant. If there are more than $3 \mathrm{~B}$ lines in a section, this is called a $\mathrm{B}+$ finding (- Fig. 1d). Direct visualization of consolidation (- Fig. 1e), pleural thickening $>3 \mathrm{~mm}$ (• Fig. 1f), and a $\mathrm{B}+$ finding are required for a diagnosis of pneumonia.

Lung computed tomography was accepted as the gold standard in the diagnosis of patients. Lung computed tomography was subsequently applied to the patients due to suspected pneumonia. Lung computed tomography results were interpreted by the radiologists working at the hospital. Lung ultrasound and lung computed tomography findings of the patients were then compared. Lung ultrasound results did not affect the diagnosis and treatment process of the patients. We evaluated the LUS results observationally.

Every patient diagnosed with viral pneumonia by CT was hospitalized. According to the guidelines of the Ministry of Health of the Republic of Turkey, patients who had respiratory rate $>30$, $\mathrm{SPO}_{2}<90$ and $\mathrm{PaO}_{2}<70$ despite $\mathrm{O}_{2}$ therapy, and had hypotension, acute kidney damage, high troponin, and lactate $>2 \mathrm{mmol}$ were followed up in the intensive care unit (ICU). Patients diagnosed with viral pneumonia based on CT results received treatment in the pandemic period even if their Reverse transcription polymerase chain reaction (RT-PCR) tests were negative.

All the data were analyzed using IBM SPSS for Windows version 25.0 (IBM Corp., Armonk, NY, USA) and MedCalc Statistical Software version 15.8 (MedCalc Software bv, Ostend, Belgium). In addition to the descriptive statistics (frequency, percentage, mean, standard deviation, median, min-max), the chi-square $\left(\mathrm{X}^{2}\right)$ test (Yates correction, Fisher's exact test) was used to compare the qualitative data. The consistency of the data to normal distribution was evaluated using the Kolmogorov-Smirnov and Shapiro-Wilk tests. An independent samples t-test was used to compare the normal distribution data. The sensitivity, specificity, and positive and negative predictive values were calculated using the

Notfall Rettungsmed 2021 · 24 (Suppl 1):S15-S20 https://doi.org/10.1007/s10049-020-00807-3 (c) Springer Medizin Verlag GmbH, ein Teil von Springer Nature 2020

\section{İ. Şan · B. Bekgöz · E. Usul · Ç. Yıldııım · E. Gemcioğlu • A. F. Kahraman · A. E. Ay \\ Role of lung ultrasonography in the diagnosis of COVID-19 patients admitted to the emergency department}

\section{Abstract}

Introduction. In this study, the use of lung ultrasonography (LUS) to diagnosis lung findings was evaluated in patients with suspected COVID-19 who were admitted to the emergency department (ED).

Methods. This observational clinical study was conducted in the ED of the Ankara City Hospital during the period April 1-30, 2020. Patients who were admitted to the ED were triaged as COVID-19 infected and who agreed to undergo LUS/LCT (lung computed tomography) were included in the study. Results. Included in the study were 40 patients who had been prediagnosed with COVID-19. Pneumonia was detected with LCT in $32(80 \%)$ patients, while the LUS examination identified pneumonia in 23 patients. The most common finding in LCT was ground-glass opacity $(n=29$, $90.6 \%)$. Of the 23 patients with pneumonia findings in LUS, 15 (65.2\%) had direct consolidation. Among the 32 patients who were found to have pneumonia as a result of LCT, 20 (62.5\%) had signs of pneumonia on LUS examination, and 12 had no signs of pneumonia. In addition, 3 patients showed no signs of pneumonia with $L C T$, but they were misdiagnosed with pneumonia by LUS. The sensitivity of LUS in the diagnosis of pneumonia in the COVID-19 patients was $62.5 \%$, while its specificity was $62.5 \%$. In addition, its positive predictive value was $87.0 \%$, and its negative predictive value was $29.4 \%$.

Conclusion. LUS may also be used in the diagnosis of pneumonia in COVID-19 patients because it is a valuable and accessible bedside diagnostic tool.

\section{Keywords}

SARS-CoV-2 - Diagnostic ultrasound . Pneumonia - Emergency service, hospital . Consolidation

\section{Rolle des Lungenultraschalls bei der COVID-19-Diagnostik in der Notaufnahme}

\section{Zusammenfassung}

Einführung. In dieser Studie wurde die Verwendung einer Lungenultraschalluntersuchung (LUS) zur Diagnose von Lungenbefunden bei Patienten, die mit Verdacht auf "coronavirus disease 19 " (COVID-19) in die Notaufnahme (NA) aufgenommen worden sind, bewertet. Methoden. Diese klinische Beobachtungsstudie wurde in der Notaufnahme des Stadtkrankenhaus Ankara in einem Zeitraum vom 1. bis 30. April 2020 durchgeführt. Patienten, die in der Notaufnahme als COVID-19-Infizierte erst eingeschätzt und Einwilligung für eine Lungenultraschalluntersuchung (LUS)/Lungencomputertomographie (LCT) gegeben haben, wurde in die Studie aufgenommen.

Ergebnisse. Insgesamt 40 mit COVID-19 vordiagnostizierte Patienten wurden in die Studie eingeschlossen. Bei 32 Patienten $(80 \%)$ wurde anhand der CT eine Pneumonie festgestellt, während die LUS bei 23 Patienten eine Pneumonie feststellen konnte. Der häufigste Befund in der LCT war eine Milchglastrübung ( $n=29,90,6 \%)$. Von den 23 Patienten mit Pneumoniebefund im LUS hatten 15 (65,2\%) eine direkte Konsolidierung. Von den 32 Patienten, bei denen die LCT einen Pneumoniebefund ergeben hatte, wiesen 20 (62,5\%) Anzeichen einer Pneumonie im LUS auf, bei 12 war dies nicht der Fall. Drei Patienten hatten keine Anzeichen einer Pneumonie in der LCT, wurden aber aufgrund der LUS mit einer Pneumonie fehldiagnostiziert. Die Sensitivität des LUS für die Diagnose einer Pneumonie bei COVID-19-Patienten betrug $62,5 \%$, die Spezifität $62,5 \%$. Der positive prädiktiver Wert betrug $87,0 \%$, der negative prädiktiver Wert $29,4 \%$.

Schlussfolgerungen. Der LUS kann auch in der Diagnostik einer Pneumonie bei COVID19-Patienten eingesetzt werden, weil er ein wertvolles und zugängliches diagnostisches Instrument am Krankenbett ist.

Schlüsselwörter

SARS-CoV-2 - Diagnostischer Ultraschall · Pneumonie $\cdot$ Notaufnahme $\cdot$ Konsolidierung 


\section{Originalien}

Table 1 Demographic characteristics and test-imaging results of patients

\begin{tabular}{ll|l|l}
\hline & & $\boldsymbol{n}=\mathbf{4 0}$ & $\mathbf{\%}$ \\
\hline Gender & Female & 20 & 50.0 \\
\hline \multirow{3}{*}{ Complaints on arrival } & Male & 20 & 50.0 \\
\hline PCR result for COVID-19 & Yes & 37 & 92.5 \\
& No & 3 & 7.5 \\
\hline CT findings & Positive & 29 & 72.5 \\
& Negative & 11 & 27.5 \\
\hline \multirow{3}{*}{ US findings } & Pneumonia & 32 & 80.0 \\
\hline CT computed tomography, US ultrasonography & Normal & 23 & 20.0 \\
\hline & Pneumonia & 17 & 57.5 \\
\hline
\end{tabular}

Table 2 Lung computed tomography (LCT) and lung ultrasound (LUS) findings of the patients

\begin{tabular}{|l|l|l|l|}
\hline CT findings & Pneumonia & 32 & 80.0 \\
\hline & Normal & 8 & 20.0 \\
\hline & - Ground-glass opacity & 29 & 90.7 \\
\hline & - Mosaic form & 1 & 3.1 \\
\hline & - Alveolar consolidation & 1 & 3.1 \\
\hline LUS findings & - Lober pneumonia & 1 & 3.1 \\
\hline & Normal & 17 & 42.5 \\
\hline & Pneumonia & 23 & 57.5 \\
& - Consolidation & 11 & 47.9 \\
\hline & - Bilateral B & 6 & 26.0 \\
\hline & - Air bronchogram & 4 & 17.4 \\
\hline & - Pleural thickening & 2 & 8.7
\end{tabular}

Table 3 Comparison of findings of lung computed tomography (LCT) and lung ultrasound (LUS)

\begin{tabular}{ll|ll} 
& & $\begin{array}{l}\text { LCT findings } \\
\text { Negative }\end{array}$ & Positive \\
\hline LUS findings & Negative & $5(62.5 \%)$ & $12(37.5 \%)$ \\
& Positive & $3(37.5 \%)$ & $20(62.5 \%)$
\end{tabular}

$\mathrm{X}^{2}$ table design. Statistical significance was set at $p<0.05$.

\section{Results}

Among the patients who were admitted to the Ankara City Hospital Emergency Department, 40 patients who had been prediagnosed with COVID-19 were included in the study. There were 20 (50\%) men and 20 (50\%) women. The mean age of the patients was $43.8 \pm 16.6$ years. While $37(92.5 \%)$ of the patients had COVID-19-related symptoms, the most common complaint was cough $(n=23,57.5 \%)$. Reverse transcription polymerase chain reaction results of 29 tients showed no signs of pneumonia with LCT, but they were misdiagnosed with pneumonia by LUS (• Table 3 ). The sensitivity of LUS in the diagnosis of pneumonia in the COVID-19 patients was $62.5 \%(95 \%$ confidence interval [CI] 43.7-78.9), while its specificity was 62.5\% (95\% CI 24.5-91.5). In addition, its positive predictive value was $87.0 \%$ (95\% CI 66.4-97.2), and its negative predictive value was $29.4 \%$ (95\% CI 10.3-56.0). The accuracy rate of LUS in detecting the pneumonia was $62.5 \%$.

Of the patients who were found to have COVID-19 pneumonia with LCT $65.6 \%$ were also COVID-19 positive according to the RT-PCR test results. Reverse transcription polymerase chain reaction results of 11 patients whose LCT results were compatible with COVID-19 were negative. LCTs Lung computed tomographies of 8 patients with COVID-19 positive RT-PCR results did not have an appearance compatible with COVID-19 (Supplementary digital content).

Pneumonia in both lungs was detected in 22 patients by LCT, while LUS revealed that $12(54.5 \%)$ of these 22 patients had bilateral pneumonia findings. While consolidation in the right lung was detected in 6 patients in LCT, $4(66.6 \%)$ of these patients had the same finding with LUS. In addition, $1(25.0 \%)$ of the 4 patients found to have consolidation in the left lung with LCT had the same finding with LUS.

\section{Discussion}

Lung ultrasound is recognized as a fast, noninvasive, easily accessible tool for diagnosing lung pathologies. In this study, we evaluated the utility of LUS in diagnosing COVID-19 in patients who visited our ED. In our study, the sensitivity and specificity of LUS in the diagnosis of pneumonia in COVID-19 patients was $62.5 \%$. Its positive predictive value was $87.0 \%$, and the negative predictive value was $29.4 \%$. In contrast, one study found that the sensitivity of LUS in the diagnosis of pneumonia was $82 \%$, and its specificity was $92 \%$ [13], while another study conducted on COVID-19 patients determined the sensitivity of LUS in the diagnosis of mild pneumonia to be $68.8 \%$ and its specificity 
85.7\% [14]. The reason LUS performed on COVID-19 patients has lower sensitivity and specificity values compared to the patients with bacterial pneumonia may be that in COVID-19, lung involvement and LUS images differ from other pneumonia agents such as bacterial pneumonia. For example, the insufficient experience of emergency physicians with chronic inflammation findings such as pleural thickening and irregularities may be an important factor in low sensitivity and specificity. In addition, reasons such as the more central distribution of lesions without contacting the lung surface in COVID19, the need to scan the entire chest in order not to miss small findings, and anatomical factors may have caused the sensitivity and specificity of LUS to be low in the diagnosis of COVID-19.

In our study, the most common finding among the patients diagnosed with pneumonia in LUS was direct visualization of lung consolidation (47.9\%). In the LUS applied to COVID-19 patients in another study, B lines were detected in different patterns in all the patients [15]. Similarly, in a study by Yasukawa et al., the most common pneumonia finding was B lines [16]. As stated in "The 2019-2020 Novel Coronavirus (Severe Acute Respiratory Syndrome Coronavirus 2) Pandemic: A Joint American College of Academic International Medicine-World Academic Council of Emergency Medicine Multidisciplinary COVID-19 Working Group Consensus Paper", consolidation is likely to be a sign of severe disease [17].

Detection of localized $\mathrm{B}+$ lines indicates a diagnosis of bacterial pneumonia, while bilateral detection of these lines signifies a diagnosis of heart failure. In addition, bilateral $\mathrm{B}+$ findings are common in COVID 19 patients. Patchy $\mathrm{B}+$ lines rather than diffuse $\mathrm{B}+$ lines in the LUS should suggest the diagnosis of COVID19 rather than lung congestion [18]. Interpretation of LUS findings supported by clinical findings will increase the rate of accurate diagnosis. In studies where LUS was applied to COVID-19 patients, the patients were diagnosed with pneumonia despite bilateral B+ findings [14-16]. Although $15 \%$ of our patients had bilateral B+findings, these patients were diag- nosed with pneumonia by the physicians who performed the LUS. We think that it is important for the LUS practitioner to know the anamnesis and physical examinations of the patients. To our thinking, the patients' prediagnoses with COVID19 may have led the physicians to decide on this diagnosis. We believe that it is more appropriate to diagnose pneumonia if bilateral $\mathrm{B}+$ findings are detected in patients who have been prediagnosed with COVID-19.

The accuracy rate of LUS in detecting the pneumonia was $62.5 \%$. According to a study [12], the diagnostic accuracy rate of LUS applied to patients with shortness of breath is $90.5 \%$. This study was conducted both with non-COVID-19 patients and in the intensive care unit. We think that the reason for the lower diagnostic accuracy rate in our study may be due to the fact that the evaluation of patients with suspected COVID-19 was performed in ED, under difficult conditions, and under personal protective clothing which altogether may have hampered accessibility of relevant regions of the chest, especially dorsobasal.

In our study, 10 of 23 patients diagnosed with pneumonia were treated in ICU. Seven of these 11 patients had consolidation in their LUS $[17,18]$. In the literature, it has been mentioned that COVID-19 may have a more severe progression if the finding of consolidation is detected. We think that the high number of consolidation findings in our study is related to the high number of patients and the severity of the patients. We think that the detection of consolidation in patients admitted to ED may warn emergency physicians that the disease may progress severely.

\section{Limitations}

The most important limitation of our study was the low number of patients. Studies with a larger number of patients will be better able to evaluate the benefit of using LUS in the diagnosis of pneumonia in COVID-19 patients. Another limitation was that LUS was not applied to all the COVID-19-suspected patients admitted to the ED; only those who were scheduled to have LCT received LUS. This may have led physicians to find consolidation in LUS. We did not ask the patients about the onset of symptoms. Lung ultrasound may not have detected the disease, since lung involvement has not yet begun in patients whose symptoms have just started. In our study, the fact that we were not able to perform dorsal examinations on patients may have caused us to miss LUS findings in some patients. In addition, the fact that the physicians were aware of the patients' clinical findings and that the prediagnosis was COVID-19 may have affected the decisions of the physicians who applied LUS.

\section{Conclusion}

Lung ultraosund may also be used in the diagnosis of pneumonia in COVID-19 patients because it is a valuable and accessible bedside diagnostic tool. It is also radiation-free and therefore safe for use with pregnant women. However, studies involving a large number of patients need to be performed, and LUS findings in COVID-19 patients need to be standardized.

\section{Corresponding address}

\section{Burak Bekgöz, MD}

Department of Emergency Medicine, Ankara City Hospital

Ankara, Turkey

drburakbekgoz@gmail.com

\section{Compliance with ethical guidelines}

Conflict of interest. I. Şan, B. Bekgöz, E. Usul, Ç. Yıldııım, E. Gemcioğlu, A.F. Kahraman and A.E. Ay declare that they have no competing interests.

This observational clinical study was conducted in the ED of the Ankara City Hospital, which was a pandemic hospital during the period April 1-30, 2020. The study was approved by the Ethics Committee No. 1 of the Ankara City Hospital (approval number E1-20-616).

\section{References}

1. https://www.who.int/docs/default-source/ coronaviruse/situation-reports/20200121-sitrep1-2019-ncov.pdf?sfursn=20a99c10_4. Accessed 16 June 2020

2. Backer JA, Klinkenberg D, Wallinga J (2020) Incubation period of 2019 novel coronavirus 
(2019-nCoV) infections among travellers from Wuhan, China, 20-28 January 2020. Euro Surveill. https://doi.org/10.2807/1560-7917.ES.2020.25.5. 2000062

3. (2005) emergency-committee-regarding-theoutbreak-of-novel-coronavirus-(2019-ncov). https://www.who.int/news-room/detail/30-012020-statement-on-the-second-meeting-ofthe-international-health-regulations-. Accessed 16 June 2020

4. https://www.who.int/docs/default-source/ coronaviruse/situation-reports/20200211-sitrep22-ncov.pdf?sfvrsn=fb6d49b1_2. Accessed 17 June 2020

5. World Health Organization (2020) Guidance W. Clinical management of severe acute respiratory infection when novel coronavirus (2019$\mathrm{nCoV}$ ) infection is suspected. WHO/2019-nCoV/ clinical/2020.5

6. Munster VJ, Koopmans M, van Doremalen N et al (2020) A Novel Coronavirus Emerging in China-Key Questions for Impact Assessment. NEngl J Med 382(8):692-694

7. Zanobetti M, Poggioni C, Pini R (2011) Can chest ultrasonography replace standard chest radiography for evaluation of acute dyspnea in the ED? Chest 139:1140-1147

8. Ai T, Yang Z, Hou H, et al (2020) Correlation of Chest CT and RT-PCR Testing in Coronavirus Disease 2019 (COVID-19) in China: A Report of 1014 Cases. Radiology 200642.

9. Radiology ACR (2020) ACR recommendations for the use of chest radiography and computed tomography (CT) for suspected COVID-19. Infection. https://www.acr.org/Advocacy-and-Economics/ ACR-Position-Statements/Recommendationsfor-Chest-Radiography-and-CT-for-SuspectedCOVID19-Infection. Accessed 20 June 2020

10. Cortellaro F, Colombo S, Coen D et al (2012) Lung ultrasound is an accurate diagnostic tool for the diagnosis of pneumonia in the emergency department. Emerg Med J 29(1):19-23

11. CardinaleL, VolpicelliG,BinelloFetal(2009)Clinical application of lung ultrasound in patients with acute dyspnea: differential diagnosis between cardiogenicand pulmonary causes. Radiol Med 114:1053-1064

12. Lichtenstein DA, Mezière GA (2008) Relevance of lung ultrasound in the diagnosis of acute respiratory failure: the BLUE protocol. Chest 134:117-125

13. Bekgoz B, Kilicaslan I, Bildik F et al (2019) BLUE Protocol Ultrasonography in Emergency Department Patients Presenting With Acute Dyspnea. Am JEmerg Med 37(11):2020-2027

14. Lu W, Zhang S, Chen B et al (2020) A Clinical Study of Noninvasive Assessment of Lung Lesions in Patients With Coronavirus Disease-19 (COVID19) by Bedside Ultrasound. Ultraschall Med 41(3):300-307

15. Lomoro P, Verde F, Zerboni F, et al (2020) COVID19 Pneumonia Manifestations at the Admission on Chest Ultrasound, Radiographs, and CT: SingleCenter Study and Comprehensive Radiologic Literature Review. Eur J Radiol Open 7:100231.

16. Yasukawa K, Minami T (2020) Point-of-Care Lung Ultrasound Findings in Patients With Novel Coronavirus Disease (COVID-19) Pneumonia. Am J Trop Med Hyg 102(6):1198-1202

17. Stawicki SP, Jeanmonod R, Miller AC, et al (2020) The 2019-2020 Novel Coronavirus (Severe Acute Respiratory Syndrome Coronavirus 2) Pandemic: A Joint American College of Academic International Medicine-World Academic Council of Emergency
Medicine Multidisciplinary COVID-19 Working Group Consensus Paper. J Glob Infect Dis 12(2): 47-93.

18. Gargani L , Soliman-Aboumarie H , Volpicelli $G$ (2020) Why, when, and how to use lung ultrasound during the COVID-19 pandemic: enthusiasm and caution. Eur Heart J Cardiovasc Imaging jeaa163. 